\title{
Case series of Salmonella spp. infections confirmed by culture test at the hospital clinical laboratory in L'Aquila (Italy), years 2011-2018
}

\author{
Vittoriano Torrelli, ${ }^{1}$ Valentina Cesarini, ${ }^{1}$ Elisa Di Giulio, ${ }^{2}$ Gianfranca Miconi, ${ }^{2}$ Francesco Gentile, ${ }^{2}$ \\ Alessia Rosciano, ${ }^{2}$ Anna Rita Tomei, ${ }^{2}$ Pamela Renzetti, ${ }^{1}$ Maria Scatigna ${ }^{1}$ \\ ${ }^{1}$ Department of Life, Health and Environmental Sciences, University of l'Aquila; ${ }^{2}$ San Salvatore Hospital Clinical \\ Laboratory - Local Health Office (ASL 1) of l'Aquila, Italy
}

\begin{abstract}
Summary
In the European Union between 2008 and 2017 there was a statistically significant decrease in the confirmed cases of salmonellosis. Nevertheless, it represents the first cause of food-borne outbreaks and the secondly most frequently reported zoonosis. Considering the pathogen diffusion and clinical consequences, monitoring the incidence of Salmonella spp. infections is a priority for public health. The study is aimed at describing the temporal trend of prevalence of Salmonella spp. positive tested and serotypes characteristics of the isolates by L'Aquila hospital laboratory microbiological analysis in 2011-2018, with a focus on the epidemic phase occurred from October 2013 to May 2014.

The survey is a case series report, with a retrospective design.
\end{abstract}

Correspondence: Maria Scatigna, University of 1'Aquila, Piazzale Salvatore Tommasi 1, 67100 L'Aquila - Coppito (AQ), Italy. Tel.: +39.0862.434692 - Fax: +39.0862.433425

E-mail maria.scatigna@cc.univaq.it

Key words: Salmonella spp., serogroup, biological samples, trend, outbreak.

Contributions: Torrelli V, Cesarini V and Scatigna M conceived the presented idea. Cesarini V \& Scatigna M developed the theory and performed the computations. Di Giulio E, Miconi G, Gentile F, Rosciano A, Tomei AR verified the analytical methods and contributed to data collection. Scatigna M encouraged A.B. to investigate the epidemiological aspect and supervised the findings of this work. All authors discussed the results and contributed to the final manuscript.

Conflict of interest: The authors declare no potential conflict of interest.

Funding: None.

Received for publication: 15 September 2020.

Revision received: 24 November 2020

Accepted for publication: 9 December 2020.

${ }^{\circ}$ Copyright: the Author(s), 2021

Licensee PAGEPress, Italy

Microbiologia Medica 2021; 36:9354

doi:10.4081/mm.2021.9354

This article is distributed under the terms of the Creative Commons Attribution Noncommercial License (by-nc 4.0) which permits any noncommercial use, distribution, and reproduction in any medium, provided the original author(s) and source are credited.
During the period considered, 220 infected carriers were identified, in particular 160 positive samples for Salmonella enterica serogroup B, so it was the main group causing salmonellosis in the area of L'Aquila both in the epidemic period and in the non-epidemic period. Moreover, the proportion of cases in early paediatric age (from one to five years) resulted statistically higher in the epidemic period than in the non-epidemic period. About the possible causes of the outbreak, the hypothesis of an environmental contamination seems to be plausible.

\section{Introduction}

Almost $60 \%$ of the most frequent human pathogens has an animal origin (23) and the majority of food zoonosis are caused by Campylobacter and Salmonella bacteria (10). Zoonoses are infectious diseases transmitted by animals to humans directly by contact with the animal or indirectly especially through contaminated water or food consumption (19). In the European Union (EU) between 2008 and 2017 there was a statistically significant decrease in the confirmed cases of salmonellosis (10) and, despite the constant decrease in the number of cases, salmonellosis represents the first cause of food-borne outbreaks and the second most frequently reported zoonosis. Recently, it has been estimated the overall incidence of human salmonellosis is 19.7 cases per 100,000 inhabitants each year in the general EU population (6) and 7.5 cases per 100,000 inhabitants in Italy (27).

Salmonella spp. are able to adapt to environmental conditions significantly different from their normal growth range (6). Infections caused by Salmonella spp. are distinguished in typhoid forms ( $S$. Typhi and $S$. Paratyphi, responsible for typhoid fever and enteric fever in general), in which mankind represents the only reservoir of the microorganism, and non-typhoid forms, caused by the so-called minor Salmonella (as $S$. Typhimurium and $S$. Enteritidis), responsible for clinical forms with a prevailing gastroenteric manifestation. Indeed, gastroenteritis is the most common worldwide manifestation of Salmonella infections (26). The most affected by salmonellosis are very young (in developmental age) or very old individuals and those with reduced immunological defenses (20).

Considering the pathogen diffusion and clinical consequences, monitoring the incidence of Salmonella spp. infections is a priority for public health. Epidemiological studies have been carried out in terms of surveillance of notified clinical cases (12) as well as number of strains isolated in the routine activities of hospitals laboratories (28); in particular, various studies concerning the incidence of different Salmonella serogroups (15) and their pres- 
ence in specific pathologies such as in the case of infectious enteritis (9) were conducted.

The study is aimed at describing the temporal trend of prevalence of Salmonella spp. positive tested and serotypes characteristics of the isolates by L'Aquila hospital laboratory microbiological analysis in 2011-2018, with a focus on the epidemic phase occurred from October 2013 to May 2014.

\section{Materials and Methods}

The study is designed as a retrospective analysis of case series which occurred in the period between June 2011 and July 2018. All the Salmonella spp. infection cases reported by San Salvatore L'Aquila hospital laboratory have been considered by extracting them from the electronic archive, in accordance with the legislation on the sensitive data use.

Different types of biological samples tested in the laboratory were considered: stool, urine, blood, peritoneal fluid, synovial fluid and tracheobronchial aspirate. The stool culture is carried out by sowing on a liquid medium, then GN broth (Liofilchem), Selenite broth (Liofilchem), or in Rappaport Vassiliadis Broth (Liofilchem). In case of positivity, a subculture on Salmonella/Shigella agar (Liofilchem) (or Hektoen enteric agar) (Liofilchem), and Chromatic Salmonella (Liofilchem) was carried out and the Salmonella spp. presence was detected by counting the colonies. The blood, peritoneal fluid and synovial fluid culture analysis is carried out with BD Bactec $^{\mathrm{TM}} \mathrm{FX}$ based on fluorescence detection. In case of positivity a subculture is performed on Mac Conkey agar (Liofilchem) and then the presence of Salmonella on Salmonella/Shigella agar (or Hektoen enteric agar) and Chromatic Salmonella and, again, by counting the colonies. The urine and tracheobronchial aspirate analysis were performed with the MacConkey agar medium and then, in case of positivity, performing a subculture on Salmonella/Shigella agar and on Hektoen enteric agar (followed by the colonies counting). The serogroup based on "O" antigenes identification consisted on an agglutination test performed by means of Wellcolex ${ }^{\mathrm{TM}}$ Colour Salmonella by Oxoid TSMX7828B (32).

The software STATA/SE 14.2 was used to perform all data analyses. The Chi-squared test and Fisher's Exact Test were applied to evaluate the significance of association between categorical variables. Measures of central tendency and of variability were used for numerical variables (arithmetic mean, standard deviation, median, inter-quartile interval), and the statistical significance of the differences was assessed using the parametric (tStudent's) and non-parametric (Kruskall-Wallis's) tests.

\section{Results}

During the entire period examined (2011-2018) an outbreak phase emerged, demonstrated by an increase in the infection cases (positive tested samples), from October 2013 to May 2014.

Table 1 shows the cases distribution, stratified in 'epidemic' and 'non-epidemic' phases: in the whole period 228 biological samples positive for Salmonella spp. were identified, from the following cultures: stool, urine, blood, tracheobronchial aspirate, peritoneal fluid and synovial fluid. Eight of these samples refer to the same patient (subsequent bacterial cultures on the same type of sample or different types of biological samples), so the total number of infected carriers identified was 220 throughout the entire period.

The differences between epidemic and non-epidemic periods are considerable: in the first period (duration of 8 months) the infected carriers were, overall, equal to 123 , with a monthly average of $15.4 \pm 7.9$ subjects; in the second period ( 77 months) they resulted equal to 97 , with a monthly average of $1.3 \pm 1.0$ subjects ( $\mathrm{p}<0.001$ to Student's t test).

Figure 1 shows the distribution of Salmonella spp. positive samples (absolute number) by year and month of acceptance of analysis requests, showing graphically the onset and duration of the epidemic peak.

Almost all positive samples detected were stool samples (213 coprocultures). Only few remaining samples were urine (7 urine cultures), blood (5 blood cultures), peritoneal fluid (1 culture), synovial fluid (1 culture) and tracheobronchial aspirate (1 culture).

Serotyping for classification based on somatic antigen "O" was performed on only for 180/228 samples (78.9\%) (Table 2) and led to the identification of:

- Salmonella enterica Group B in $88.9 \%$ of the samples (152 stool samples, 5 urine samples and 3 blood samples);

- Salmonella enterica Group C in 3,9\% of the samples (6 stool samples and in the only positive sample of synovial fluid);

Salmonella enterica Group D in 6,1\% of the samples (10 stool samples and in 1 blood sample);

Salmonella enterica Group E and Salmonella enterica Group $\mathrm{G}$ in the same stool sample, in which, moreover, a strain of Salmonella enterica from Group B was also found.

Serotyping was carried out more frequently during the epidemic period $(88.9 \%$ of the samples) than the non-epidemic period $(66.7 \%)$. Differences are found, also, in respect to serotypes identified:

- during the 2013-2014 epidemic period, almost all of typed strains, equal to $99.1 \%(111 / 112)$, were Salmonella enterica Group B (the only one that wasn't, was found belonging to the Group C serotype);

- in the non-epidemic period, the distribution was less unbalanced: $72.1 \%$ (49 stool samples out of 68 serotyped) were Group B, 16.2\% (10/58) Group D, 8.8 \% (6/68) Group C and $1.5 \%$ Groups E and G (1/68).

Positive biological samples refer, overall, to male patients for $53.5 \%$ and to women for $46.5 \%$.

Table 1. Absolute frequency of Salmonella spp. detections, no. of infected carriers, length of observational periods (in months) and average numbers of infected carriers per months, stratified by period (arithmetic mean \pm standard deviation).

\begin{tabular}{lcccc} 
& Non-epidemic period & Epidemic period & Total & P \\
No. positive samples & 102 & 126 & 228 & 220 \\
No. infected carriers & 97 & 123 & 85 & $<0.001$ \\
\hline Duration (months) & 77 & 8 & $2.6 \pm 4.8$ & \\
Average carriers/month & $1.3 \pm 1.0$ & $15.4 \pm 7.9$ &
\end{tabular}


The chronological age varies from a minimum value of 0 to a maximum value of 93.1 years.

Figure 2 shows the median value and the interquartile range of the age per year of detection and highlights the strong variability of these values over the observed time period: in the years of the outbreak (2013 and 2014) the lowest values are recorded and these differences were statistically significant at the Kruskall-Wallis non-parametric test $(\mathrm{p}<0.001)$.

This trend is confirmed by the distribution per age class and survey year shown by Figure 3 stratified by period (epidemic vs non-epidemic):

- the proportion of carriers in early pediatric age is statistically higher in the period of the outbreak 2013 - 2014: for the 'less than 1 year' group it is equal to $4.8 \%$ of cases in the epidemic period and $2.0 \%$ of cases in the non-epidemic period; for the ' $1-2$ ' years group, respectively, $34.9 \%$ vs $5.9 \%$;

- for the higher age groups, the differences are reversed: in that ' $5-18$ ' years they are respectively equal to $18.3 \%$ of the cases of the epidemic period and to $25.5 \%$ of the cases of the nonepidemic period; in that ' $18-64$ ' years the proportions of sub- jects are, respectively, $9.5 \%$ vs $19.6 \%$ and, lastly, for the ' 65 years and more', respectively $10.3 \%$ vs $22.5 \%$ ( $<<0.001$ at the Chi Square test).

\section{Discussion}

From a public health perspective, one point of strength of this study is the continuative period of observation (from 2011 to 2018). A fairly constant level of human infections of pathogen Salmonella spp. was confirmed, except for the epidemic period (2013-2014). In the EU, Salmonella is the most frequently etiological agent detected in food-borne outbreaks. The most recent report by EFSA and ECDC reported a notification rate in the EU of 19.7 cases of salmonellosis per 100,000 population, a slightly lower rate (2.9\% decrease) compared to 2016 (20.4 cases per 100,000 population) (10). As in the previous year, Italy was among the countries that recorded the lowest rates ( $<7.0$ cases per 100,000 population). This confirms the decreasing trend of human infections recorded

Table 2. Stratification of the strains by the ' $O$ ' antigen serotyping.

\begin{tabular}{|c|c|c|c|c|c|c|}
\hline & & & Non- & eriod & & \\
\hline & $\mathrm{N}^{2}$ & $\%$ & $\mathbf{N}$ & $\%$ & $\mathbf{N}$ & $\%$ \\
\hline S. enterica Group B & 111 & 99.1 & 49 & 72.1 & 160 & 88.9 \\
\hline S. enterica Group C & 1 & 0.9 & 6 & 8.8 & 7 & 3.9 \\
\hline S. enterica Group D & 0 & 0.0 & 11 & 16.2 & 11 & 6.1 \\
\hline S. enterica Group E & 0 & 0.0 & 1 & 1.5 & 1 & 0.6 \\
\hline S. enterica Group G & 0 & 0.0 & 1 & 1.5 & 1 & 0.6 \\
\hline Total & 112 & 100 & 68 & 100 & 180 & 100 \\
\hline
\end{tabular}

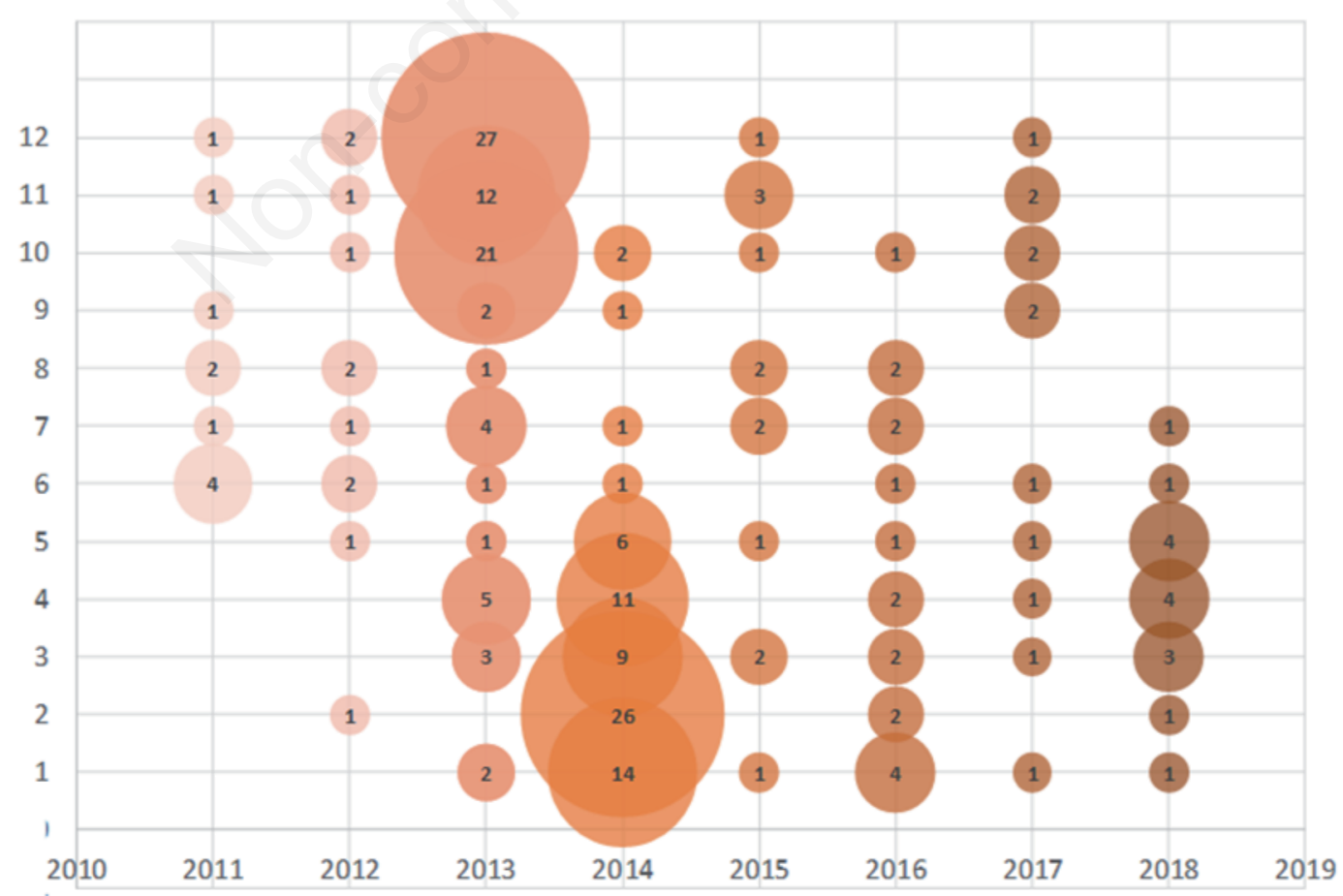

Figure 1. Distribution of Salmonella spp. positive samples, by year and month of acceptance of the analysis requests. 
since the nineties at global (17) and Italian level (5). The source of our data (analytical findings reported at the hospital laboratory) does not allow us to calculate the accurate incidence of the infection since they do not precisely match the notifications of human disease in the area of interest. Nevertheless, the number of human carriers of Salmonella spp. in terms of positive biological samples during the non-epidemic phase (1.3 carriers/months in average), compared to the population denominator of geographical area served by the hospital laboratory (circa 100,000 inhabitant) is 15.6 carriers/100,000 per year, so consistent with the epidemiological outline of Salmonellosis at European level but higher compared to the Italian incidence. During the epidemic period (2013-2014 outbreak) the value of 184.8 carriers/100,000 inhabitants was reached, so, more than tenfold.

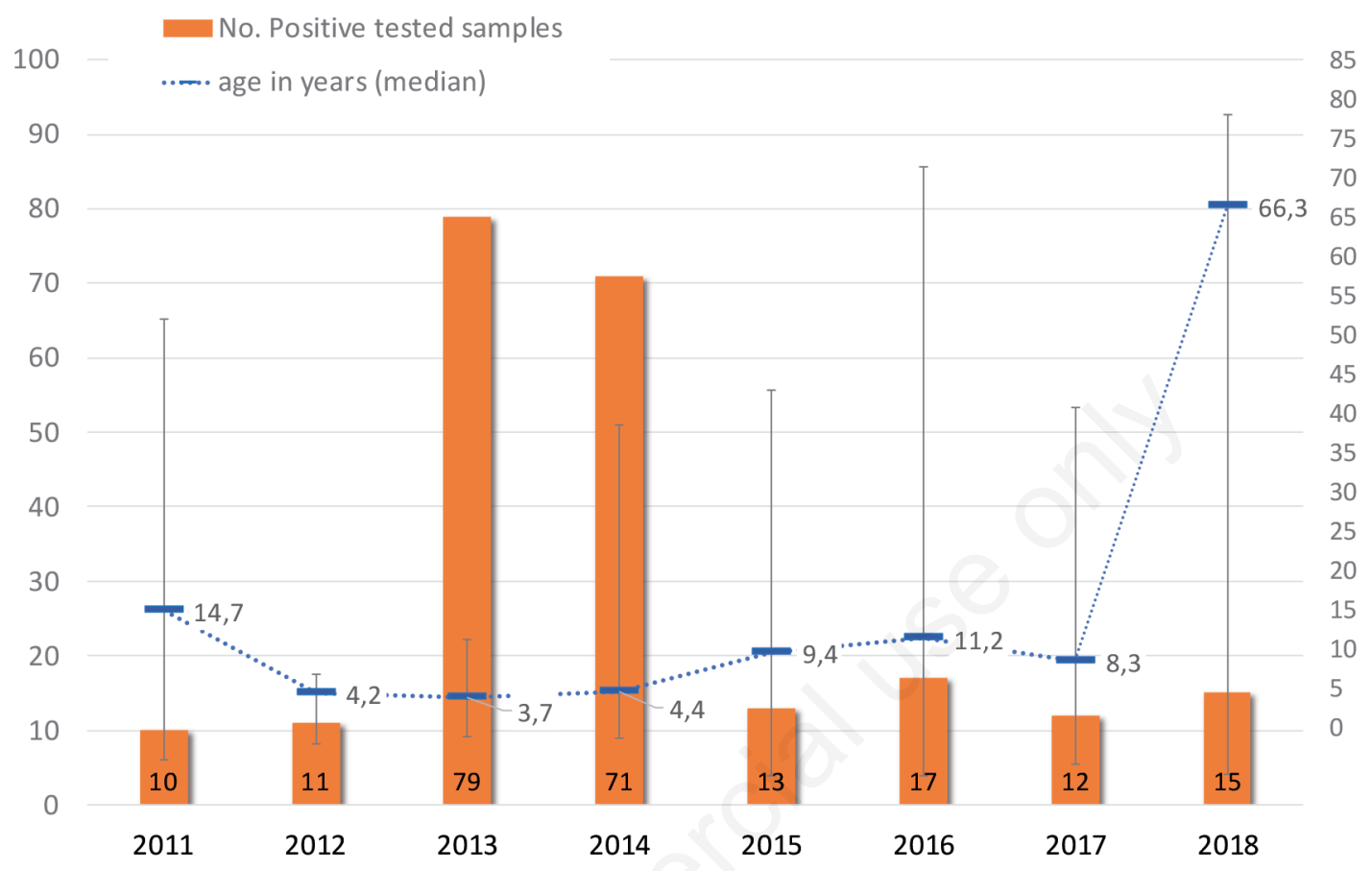

Figure 2. Number of positive samples per year of investigation and chronological age of infected carriers expressed as median value and interquartile range.

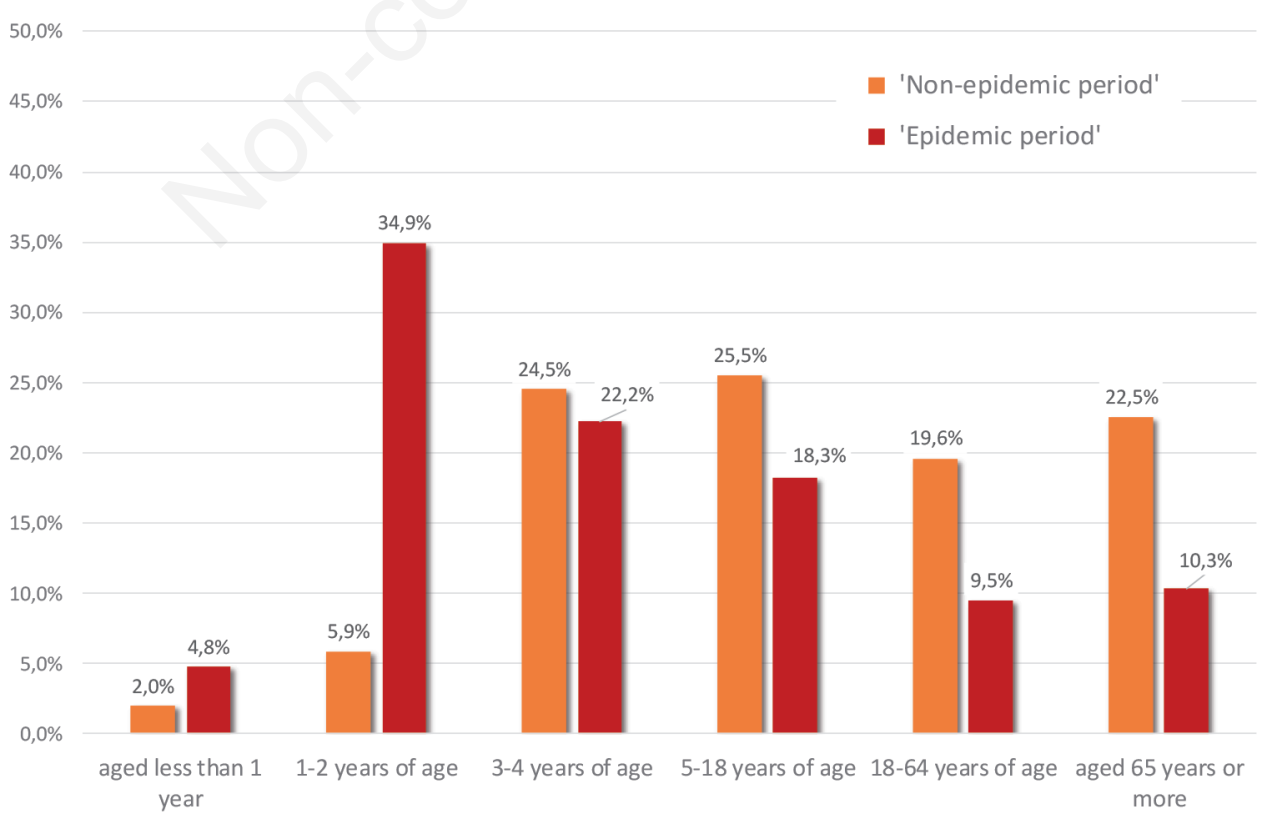

Figure 3. Distribution of identified subjects positive for Salmonella spp. by age group in the 'epidemic period' and in the 'non-epidemic' period. 
Food contamination can occur at any point in the supply chain, from 'farm to fork'; it can, indeed, occur during production, collection, processing, storage, transport, retail sale and domestic handling (30). The food products mainly attributed to the onset of the infection are animal origin products such as milk, poultry and eggs. So, the implementation of new company control measures of Salmonella infection in food companies, such as the introduction of live vaccines in poultry, the improvement of hygiene and the consumers and workers education of the food industry has proved effective (17).

Notification of non-typhoidal salmonellosis in humans is mandatory in most EU member States. The diagnosis of infection is generally made by culture from human stool samples. Almost all the samples we analyzed showing positivity are faecal samples, just like the national data reporting the faecal biological matrix as the prevailing isolation matrix (21). Almost all member States, except Bulgaria, perform serotyping of the isolates. As in previous years, the three Salmonella serovars most commonly reported in 2017 were $S$. Enteritidis, $S$. Typhimurium and $S$. Typhimurium monophasic variant (belonging to the enterica subspecies) (14); these three serovars accounted for $78.1 \%$ of confirmed human cases acquired in the EU (10). According to European data, the American CDC reported that among the 20 serotypes most frequently associated with Salmonella spp. appear $S$. Enteritidis and $S$. Typhimurium (30). Most serovars show a marked seasonality, with an increase in cases during the summer months (with a peak in August/September), which may be related to the parallel trend of Salmonella spread in animal hosts and/or insufficient refrigeration and poor management of food during the warm months (29).

During the time observed there was an epidemic phase lasting from October 2013 to May 2014, during which the number of positive samples exceeded 10 per month. These levels are clearly in excess of those of previous and subsequent years. For example, between 2005 and 2009, the average number of cases of salmonellosis reported in the province of L'Aquila amounted to nine per year, with a maximum value of 30 annual cases in 2005 (8). The epidemic phase has been confirmed and described in two previous analyzes. Cito et al., following an alert sent to the public health services of Abruzzo region, conducted a survey detecting cases from January 2013 to October 2014 (8). According to our data, the epidemic period occurred from June 2013 to May 2014, period in which cases were continuously detected by hospitals in the province. Our analyzes showed that the proportion of cases in early pediatric age (from one to five years) is statistically higher in the epidemic period than in the non-epidemic period, a figure confirmed by both analyzes. In fact, in the Cito et al. survey, individuals under the age of four years are the most affected (8). Nigro et al. conducted the survey analyzing only three months of the epidemic period (October-December 2013) and focusing on individuals aged between one and fifteen years (29). In this sample population, the age group most at risk that of children in early childhood, with a greater severity of symptoms in children under two years of age. This is consistent with what other studies have shown, i.e. younger children are more at risk of infection (17). In a British study, the highest reported incidence of the disease is in children younger than 4 years and it decreases in the older age groups up to 14 years (25). A possible explanation is that in younger children there is a higher percentage of symptomatic infections with a consequent greater probability that the parents take the children to the doctor and that the doctors require analysis and tests (13), even if this hypothesis is refuted in some other studies (29).

Nigro et al. believe that the greatest susceptibility may depend on behavioral factors related to age and the increased exposure to the vehicle of infection in the domestic environment due to cross-contamination, especially when the vehicle is a food for infants (29).

No information is available in the literature to explain the very high median age of 2018 cases compared to other years. It could be supposed that the reports belong to old people living in retirement homes. Indeed, human Salmonella infections frequently involve several subjects at the same time, especially if they live together (such as retirement homes) and if they have had access to the same food sources (such as canteens) (1). Even when standards of care and food hygiene are very high some infections are inevitable because of susceptibility of the population and the highly infectious and persistent nature of enteric pathogens (24). Gastroenteritis is quite common in retirement homes and can occur in sporadic or epidemic form due to viral agents, but bacterial forms are also possible, especially from Salmonella spp., E. coli, Clostridium difficile (3).

In our study the most identified Salmonella spp. serogroup was Group B, both in the epidemic and in the non-epidemic period. In the previous investigations (Cito et al.; Nigro et al.), conducted on the data of the epidemic period, S. Typhimurium monophasic variant was indicated as the strain responsible for the outbreak, and this confirms our serological investigation for the epidemic period. That strain belongs to Group B (33) and this has been the most reported serovar in Italy since 2000 (17). However, this data counteracts the European ones. Fuche et al. reported in Europe in 2012, $42 \%$ of cases belonged to serogroup D followed by B (32.7\%) and C $(8.4 \%)$ serogroups (15). In fact, $S$. Enteritidis belongs to serogroup D (30) which was the mainly acquired serovar in Europe in 2017 , almost two out of three $(61.2 \%)$ of the reported cases were infected with $S$. Enteritidis (10).

Nonetheless, the environmental occurrence of Salmonella Group B in Italy is documented by literature. $S$. Typhimurium and Monophasic $S$. Typhimurium represent the first two serotypes isolated from veterinary matrices (animals and food) in Italy. This is a characteristic of our country, though generally in Europe the most isolated serotype remains $S$. Enteritidis (22). Confirming this, Gargiulo et al. (16) found Salmonella Group B in birds of prey, Chiari et al. (7) in wild boars and Botti et al. (2) in wild mammals and birds. In all of these investigations the most common isolated serovars was $S$. Typhimurium. Nevertheless, there are surveys which indicate that the frequent presence of Monophasic S. Typhimurium is not a phenomenon limited only to Italy. Helmuth et al. reported in Denmark an outbreak of monophasic $S$. Typhimurium from October 2018 to January 2019; the most likely vehicle was medister sausage (18).

Carraturo et al. define the prevalence of Salmonella enterica in the final stage of the meat production chain, on samples collected from slaughterhouses in Italy; the most isolated serovar was $S$. Choleraesuis (62\%) (4).

Perugini et al. isolated Salmonella enterica strains Typhimurium and Enteritidis in Italy from animals and food such as bovine, ovine, swine and chicken meat, shellfish, eggs, bovine ad bubaline milk (31).

These data underline the existence of an environmental circulation of this serogroup, confirming the hypothesis that during the epidemic period the greater circulation can be ascribed to a natural origin, i.e. from zoonotic reservoirs that have contaminated the environment (land and then surface waters). While during the nonepidemic period the infection could have been caused by the consumption of contaminated food from other countries, i.e. eggs from other European countries. As matter of fact, a multi-country outbreak of $S$. Enteritidis, linked to eggs from Poland, has been ongoing in the EU/EEA for several years (11).

The presence of Salmonella enterica from Group B in a stool sample were Salmonella enterica Group E and Salmonella enterica Group $\mathrm{G}$ were found, so, having three different serogroups in the same specimen, could be indicative of sample contamination. 
Therefore, about the possible causes of the outbreak, the hypothesis of an environmental contamination seems to be plausible and supported by the identification, in other regions, of clinical cases due to the same strain only in patients who had made a trip to L'Aquila (29). Environmental contamination could result from animal sources. In the Salmonella epidemiological cycle, the animals act as main maintenance reservoirs. Environmental contamination of soil, surface waters, work environments, can promote the spread and contact of bacteria with receptive animal species and with humans (20). Indeed, there may be a correlation between the copious rainfall before the onset of the outbreak and the presence of Salmonella spp. in surface waters. Contamination of these waters may have occurred due to sewage water flooding because of heavy rainfall and there may have been problems with sewage treatment plants related to the persistent damage caused by the devastating earthquake in L'Aquila in 2009 (8).

\section{Conclusions}

In this survey we described the epidemiological trend of Salmonella spp. infections detected by microbiological analysis of human samples from June 2011 to October 2018 at the San Salvatore L'Aquila hospital microbiological analysis laboratory. In particular, the study evaluated the temporal trend of Salmonella spp. found during the observation period and serological characteristics of isolated strains.

The findings support and integrate previous studies on the same outbreak which considered only the hospitalized paediatric cases (Nigro et al, 2016). The difference in patient's age between epidemic and non-epidemic phases confirmed the high proportion of young patients involved, as well as the unusual feature of pathogen dissemination during the outbreak, with higher epidemiological impact and different serogroup distribution. Our study highlights the importance of the collaboration between health service laboratories and the public health sector, in the perspective of epidemiological surveillance at the local level.

\section{References}

1. Azienda Sanitaria Universitaria Friuli Centrale (ASUFC). Il controllo delle infezioni nelle strutture residenziali sociosanitarie - Manuale operativo (Editors: Mantoan P., Mantoan P., Pellizzer G., Cazzaro R., Fedeli U., Spolaore P.) (2017). http://www.aas3.sanita.fvg.it/it/azienda_informa/whatsaas3/17 1826 salmonella.html.

2. Botti V, Navillod FV, Domenis L, et al. Salmonella spp. and antibiotic-resistant strains in wild mammals and birds in northwestern Italy from 2002 to 2010. Vet Ital 2013;49:195-202.

3. Buonfrate D, Mantoan P, Pellizzer G, et al. Il controllo delle infezioni nelle strutture residenziali sociosanitarie. Manuale operativo. Ccm-network 2007: http:/www.ccm-network.it/documenti_Ccm/prg_area1/Inf_Oss/Regioni/Veneto_manuale_infe zioni.pdf.

4. Carraturo F, Gargiulo G, Giorgio A, et al. Prevalence, Distribution, and Diversity of Salmonella spp. in Meat Samples Collected from Italian Slaughterhouses. J Food Sci 2016;21:M2545-51.

5. Carrieri MP, Salmaso S, Bella A, et al. Evaluation of the SIMI system, an experimental computerised network for the surveillance of communicable diseases in Italy. Eur J Epidemiol 2000;16:941-7.
6. Chlebicz A, Śliżewska K. Campylobacteriosis, Salmonellosis, Yersiniosis, and Listeriosis as Zoonotic Foodborne Diseases: A Review. Int J Environ Res Public Health 2018;15:863.

7. Chiari M, Zanoni M, Tagliabue S, et al. Salmonella serotypes in wild boars (Sus scrofa) hunted in northern Italy. Acta Vet Scandin 2013;55.

8. Cito F, Baldinelli F, Calistri P, et al. Outbreak of unusual Salmonella enterica serovar Typhimurium monophasic variant 1,4 [5],12: i: Italy, June 2013 to September 2014. Euro Surveill 2016;21(15).

9. Distasi MA, Porzio M, Del Gaudio T. Epidemiology of enteric pathogens found in stool symptomatic patients selected in a northern Bari region population between 2000 and 2009. Microbiol Med 2011;26:2362.

10. EFSA and ECDC. The European Union summary report on trends and sources of zoonoses, zoonotic agents and foodborne outbreaks in 2017. EFSA J 2018;16:5500.

11. EFSA and ECDC. Multi-country outbreak of Salmonella Enteritidis infections linked to eggs, third update. 2020. doi: https://doi.org/10.2903/sp.efsa.

12. Eng SK, Pusparajah P, Ab Mutalib NS, et al. Salmonella: A review on pathogenesis, epidemiology and antibiotic resistance. Front Life Sci 2015;8:284-93.

13. European Centre for Disease Prevention and Control (ECDC). Salmonellosis. In: ECDC. Annual epidemiological report for 2016. Stockholm: ECDC; 2019.

14. Ferretto. Sistema delle MALattie INFettive (malinf). Available from: http://www.malinf.com/batteria-index/Salmonella.html accessed on 15 Sept 2020.

15. Fuche FJ, Sow O, Simon R, Tennant SM. Salmonella Serogroup C: Current Status of Vaccines and Why They Are Needed. Clin Vacc Immunol 2016;23:737-45.

16. Gargiulo A, Fioretti A, Russo TP, et al. Occurrence of enteropathogenic bacteria in birds of prey in Italy. Lett Appl Microbiol 2017;66:202-6.

17. Graziani C, Mughini-Gras L, Owczarek S, et al. Distribution of Salmonella enterica isolates from human cases in Italy, 1980 to 2011. Euro Surveill 2013;18:20519.

18. Helmuth IG, Espenhain L, Ethelberg S, et al. An outbreak of monophasic Salmonella Typhimurium associated with raw pork sausage and other pork products, Denmark 2018-19. Epidemiol Infect 2019;147:1-7.

19. Istituto Superiore di Sanità. Epicentro (ISS, Epicentro). Il portale dell'epidemiologia per la sanità pubblica. https://www.epicentro.iss.it/zoonosi/ accessed on 15 Sept 2020.

20. Istituto Superiore di Sanità. Salmonella infections: diagnosis, epidemiology and surveillance. Graziani C, Galetta P, Busani L, Dionisi AM, Filetici E, Ricci A, Caprioli A, Luzzi I (Editors). 2005, 49 p. Rapporti ISTISAN 05/27.

21. Istituto Zooprofilattico delle Regioni Lazio e Toscana (IZSLT). Rapporto regionale sulla sorveglianza di laboratorio - Anno 2010 (Editors: Tolli R, Greco S, Di Giampietro G, Marrocco MG, Vita S, Bilei S). Quaderni di Zooprofilassi. 2012; 8. http:// www.izslt.it/wp-content/uploads/2015/06/Quaderni_zooprofilassi_Febbraio_2012.pdf.

22. Istituto Zooprofilattico Sperimentale delle Venezie (IZSVe). Sierotipi emergenti di Salmonella, il successo epidemiologico della variante monofasica di S. Typhimurium. (2018). Tratto il giorno 11 20, $2020 \mathrm{https} / /$ www.izsvenezie.it/sierotipi-emergenti-salmonella-variante-monofasica-styphimurium/.

23. Karesh WB, Dobson A, Lloyd-Smith JO, et al. Ecology of zoonoses: natural and unnatural histories. Lancet 2012;380: 1936-45.

24. Kirk MD, Roberts L, Horvath J. Foodborne and Waterborne 
Infections in Elderly Community and Long-Term Care Facility Residents, Victoria, Australia. Emerg Infect Dis 2012;18:377-84.

25. Lake IR. Food-borne disease and climate change in the United Kingdom. Environ Health 2017;16:117.

26. Majowicz SE, Musto J, Scallan E, et al. The global burden of nontyphoidal Salmonella gastroenteritis. Clin Infect Dis 2010;50:882-9.

27. Mascaro V, Pileggi C, Crinò M, et al. Non-typhoidal Salmonella in Calabria, Italy: a laboratory and patient-based survey. BMJ Open 2017;7:e017037.

28. Miseferi G, Candelieri G, Re M, et al. Infections caused by Salmonella: epidemiological investigation in Rho Hospital. Microbiol Med 2010;25:2470.

29. Nigro G, Bottone G, Maiorani D, et al. Pediatric Epidemic of Salmonella enterica Serovar Typhimurium in the Area of L'Aquila, Italy, Four Years after a Catastrophic Earthquake. Int J Environ Res Publ Health 2016;13:475.
30. Oh JH, Park MK. Recent Trends in Salmonella Outbreaks and Emerging Technology for Biocontrol of Salmonella Using Phages in Foods: A Review. J Microbiol Biotechnol 2017;27:2075-88.

31. Perugini AG, Carullo MR, Esposito A, et al. Characterization of antimicrobial resistant Salmonella enterica serovars Enteritidis and Typhimurium isolates from animal and food in Southern Italy. Vet Res Commun 2010;34:387-92.

32. Scientific ${ }^{\text {TM }}$, T. (2012, agosto). Thermo Scientific ${ }^{\text {TM }}$ Kit test di agglutinazione in lattice rapido Wellcolex ${ }^{\mathrm{TM}}$ Color Salmonella. Tratto da fisher scientific part of Thermo Fisher Scientific ${ }^{\text {TM}}$ : www.oxoid.com/ifu.

33. WHO Collaborating Centre for Reference and Research on Salmonella. ANTIGENIC FORMULAE OF THE SALMONELLA SEROVARS. 9th edition (Editors Grimont PAD \& Weill FX). 2007 Geneva. https://www.pasteur.fr/sites/default/ files/veng_0.pdf. 